\title{
The dissemination of genetic improvement through the French pig industry
}

\author{
R. GUEBLEZ, L. OLLIVIER \\ I.N.R.A., Station de Génétique quantitative et appliquée, \\ F 78350 Jouy-en-Josas
}

\begin{abstract}
Substantial genetic improvement in French breeding farms (nucleus) over the last fifteen years has been demonstrated. The question dealt with in this paper is that of the extension of this improvement to the commercial farm (base). In the 1st part of the paper, the difference in genetic level between the nucleus and the base, i.e. the improvement lag, is evaluated using a simple model of migration through a three-tier «pyramid» : nucleus, multiplier, commercial. The lag can then be expressed as a function of the proportion of genes entering commercial herds from the nucleus (a), multiplier (b) and commercial (c) levels. From a survey of the exchanges of breeding animals in France in 1978, the following values have been obtained : $a=0.43 ; b=0.23 ; c=0.34$. By also taking account of the genetic superiority of the boars migrating directly from the nucleus to the commercial farm, the estirnated lag is 1.8 generation, or 3.6 years of selection, which is equivalent to $29 \mathrm{FF}$ (1981) in overall economic value per pig fattened. In the 2nd part of the paper, an attempt is made to measure the actual lag as well as the evolution through time of the commercial level. Comparisons carried out in the testing stations between the genetic level of the purebreds and that of the commercial products confirm the existence of a genetic lag of the latter relative to the former, consistent in sign but variable in size through the period 1972-1981. On the other hand, the trends observed between 1975 and 1980 over a large sample of commercial farms, for growth rate and feed conversion, represent $2 / 3$ of the genetic trends estimated in the nucleus (over the period 1965-1973). However, as genetic comparisons are difficult to make in commercial farms, the genetic part of the trends observed cannot be established. Possible genotype-environment interactions must also be considered when dealing with trends in performances measured under commercial conditions.
\end{abstract}

\section{Choice of boar line for terminal crossing}

\author{
P. SELLIER \\ I.N.R.A., Station de Génétique quantitative et appliquée, \\ F 78350 Jouy-en-Josas
}

Pig sire lines in France are characterized by a broad variety since at the present time about ten boar lines are used to a significant extent. This diversity originates from a number of causes : the main explanation is the between-region variations in the relative economic impact of carcass quality on the benefit per pig and the market demands in terms of carcass weight and conformation.

The qualities required from the terminal boar are reviewed. The merit of a male line mainly depends on (1) the reproductive ability of the boar and its influence on the numerical ploductivity of the sow herd, (2) the sire effect on progeny production traits. Other aspects such as the «cost price» of the young boar and the expectation of future genetic improvement within the sire line are also evoked. It is to be pointed out that the many traits contributing to the overall merit of a boar line are of unequal importance according to the point of view considered.

Assuming that the commercial crossbred female proceeds from the French Landrace $\times$ Large White combination, the choice of the terminal male line offers a double alternative : (1) backcrossing or crossing with a « specialized » terminal boar, (2) purebred or crossbred 